\title{
SYMPLECTIC GENUS, MINIMAL GENUS AND DIFFEOMORPHISMS*
}

\author{
BANG-HE LI ${ }^{\dagger}$ AND TIAN-JUN LI ${ }^{\ddagger}$
}

\begin{abstract}
In this paper, the symplectic genus for any 2-dimensional class in a 4-manifold admitting a symplectic structure is introduced, and its relation with the minimal genus is studied. It is used to describe which classes in rational and irrational ruled manifolds are realized by connected symplectic surfaces. In particular, we completely determine which classes with square at least -1 in such manifolds can be represented by embedded spheres. Moreover, based on a new characterization of the action of the diffeomorphism group on the intersection forms of a rational manifold, we are able to determine the orbits of the diffeomorphism group on the set of classes represented by embedded spheres of square at least -1 in any 4 -manifold admitting a symplectic structure.
\end{abstract}

1. Introduction. Let $M$ be a smooth, closed oriented 4-manifold. An orientation-compatible symplectic form on $M$ is a closed two-form $\omega$ such that $\omega \wedge \omega$ is nowhere vanishing and agrees with the orientation. For any oriented 4-manifold $M$, its symplectic cone $\mathcal{C}_{M}$ is defined as the set of cohomology classes which are represented by orientation-compatible symplectic forms.

For any class $e \in H^{2}(M ; \mathbf{Z})$, its minimal genus $m(e)$ is the minimal genus of a smoothly embedded connected surface representing the Poincaré dual $\operatorname{PD}(e)$. The problem of determining the minimal genus has involved many of the important techniques in 4-manifold topology, and it bears its origin in the older problem of representing the Poincaré dual to a class by an embedded sphere (See the excellent survey papers [La1-2] and [Krl] on these two problems).

We are here interested in studying both these problems for 4-manifolds with non-empty symplectic cone. We will introduce the notion of the symplectic genus $\eta(e)$ for 4-manifolds with non-empty symplectic cone. Recall that any symplectic structure $\omega$ determines a homotopy class of compatible almost complex structures on the cotangent bundle, whose first Chern class is called the canonical class of $\omega$. Roughly, the symplectic genus $\eta(e)$ of a class $e$ is given by the formula $\left[e^{2}+K \cdot e\right] / 2+$ 1 , where $K$ has largest pairing against $e$ amongst canonical classes of symplectic structures for which the symplectic area of $e$ is positive.

$\eta(e)$ has many nice properties, among which are (i) invariance under the action of diffeomorphism group and (ii) bounding the minimal genus from below. We speculate that, for most class of positive square, the symplectic genus is in fact the minimal genus, at least when $b^{+}(M)=1\left(b^{+}(M)\right.$ is the maximal dimension of a positive definite subspace of $\left.H^{2}(M ; \mathbf{R})\right)$. The minimal genus, by definition, is non-negative. And it is easy to see that the symplectic genus of a sufficiently large multiple of a class of positive square is positive. However it is not obvious that the symplectic genus of any class of positive square is non-negative. In this paper we prove

Theorem A. Let $M$ be a smooth, closed oriented 4-manifold with non-empty symplectic cone and $b^{+}(M)=1$. Then the symplectic genus of any class of positive

\footnotetext{
*Received August 14, 2001; accepted for publication October 31, 2001.

† Academy of Mathematics and Systems Science, Academia Sinica, Beijing, 100080, P.R. China (libh@iss06.iss.ac.cn).

$\ddagger$ Department of Mathematics, Princeton University, Princeton, NJ 08544, USA (tli@math.princeton.edu).
} 
square is non-negative, and it coincides with the minimal genus for any sufficiently large multiple of such a class.

The proof of Theorem A is not very difficult except when the manifold is a nonminimal rational or irrational ruled manifold. In fact, for this kind of manifold we are able to obtain a much stronger result. Let us explain what such a manifold is. Let $\mathcal{E}_{M}$ be the set of integral cohomology classes whose Poincaré duals are represented by smoothly embedded spheres of squares -1 . $M$ is said to be (smoothly) minimal if $\mathcal{E}_{M}$ is the empty set. Any manifold $M$ can be decomposed as a connected sum of a minimal manifold $N$ with some number of $\overline{C P}^{2}$. Such a decomposition is called a (smooth) minimal reduction of $M$, and $N$ is a minimal model of $M . M$ is said to be rational if one of its minimal models is $C P^{2}$ or $S^{2} \times S^{2}$; and irrational ruled if one of its minimal models is an $S^{2}$-bundle over a Riemann surface of positive genus. When $M$ has non-empty symplectic cone and is not rational or irrational ruled, $M$ has a unique minimal reduction (see [L1] and [Mc3]). Using the invariance of the symplectic genus under diffeomorphisms and the Taubes-Seiberg-Witten theory, we are able to show

Theorem B. Let $M$ be a rational or irrational ruled 4-manifold. If $e$ is a class with square at least one, then its symplectic genus is non-negative and computable. Furthermore, if $e \cdot e \geq \eta(e)-1$, then $\mathrm{PD}(e)$ is represented by a connected symplectic surface, and therefore its minimal genus coincides with its symplectic genus.

For classes with square zero and -1 on rational and irrational ruled manifolds, we have similar results.

Observe that if $\operatorname{PD}(e)$ is represented by an embedded sphere, then $m(e)=0$ and therefore $\eta(e)$ is zero as well. It turns out that this simple fact enables us to completely determine which class of square at least -1 is represented by a smoothly embedded sphere in any symplectic 4 -manifold. When $M$ has nonempty $\mathcal{C}_{M}$ and is not rational or irrational ruled, such a description is known (see [T2], [Mc3] and [L1]). Let $N \# n \overline{C P^{2}}$ be the unique minimal reduction of $M$, then, if $e$ has square at least -1 , $\mathrm{PD}(e)$ is represented by a smoothly embedded sphere if and only if $e$ is a generator of one of the $\overline{C P}^{2}$. For rational and ruled manifolds, we have

Theorem C. Let $M$ be a rational or irrational ruled manifold and $e \in H^{2}(M)$ be a class with square at least -1 . If $\eta(e)=0$, then $\operatorname{PD}(e)$ is represented by a smoothly embedded sphere. Furthermore, if $\operatorname{PD}(e)$ is represented by a smoothly embedded sphere, then either $\eta(e)=0$ or $e$ is a non-primitive class of square zero with $e=p e^{\prime}$ and $\eta\left(e^{\prime}\right)=0$.

We would like to remark that the proofs of Theorems A, B and C are built out of the work Taubes on Seiberg-Witten invariants realizing symplectic surfaces, the wall crossing formula for proving the non-triviality of the Seiberg-Witten invariants, and the fact that for minimal manifolds with $b^{+}=1$ it is easy to force symplectic surfaces to be connected. For non-minimal manifolds, we need the additional techinical notion of the reduced class.

Beyond determining the set of classes represented by spheres and with square at least -1 , we are also able to determine the action of the diffeomorphism group on this set. Let us call a class spherically representable if its Poincaré dual is represented by a smoothly embedded sphere. Let $\mathcal{S P H}(M)$ be the set of spherically representable classes and $\mathcal{S P H} \mathcal{H}_{\geq-1}$ be the subset of classes with square at least -1 . Obviously $\operatorname{Diff}(M)$ acts on $\mathcal{S P H}(M)$ and preserves $\mathcal{S P H} \mathcal{H}_{\geq-1}$. We are able to completely determine the orbits of $\mathcal{S P H} \mathcal{H}_{\geq-1}$ under $\operatorname{Diff}(M)$. To state the result we need to introduce 
more notations. We say a class $e$ is of divisibility $p$ if $e=p \tilde{e}$ with $\tilde{e}$ a primitive class. Let $\mathcal{S P H} \mathcal{H}_{s, p}(M)$ be the subset of classes in $\mathcal{S P H}(M)$ with square $s$ and divisibility $p$. $\mathcal{S P} \mathcal{H}_{s, p}(M)$ can be further decomposed depending on the type, i.e. whether a class is characteristic or ordinary. Recall a class is called characteristic if it is an integral lift of the second Stieffel-Whitney class. Such a class $v$ satisfies $v \cdot u=u \cdot u(\bmod 2)$ for any class $u$. A class is called ordinary if it is not characteristic. Define $\mathcal{S P H}_{s, p}^{o}(M)$ and $\mathcal{S P \mathcal { H }} \mathcal{H}_{s, p}^{c}(M)$ to be the subsets of ordinary and characteristic classes in $\mathcal{S P H}_{s, p}(M)$. When the group of diffeomorphisms $\operatorname{Diff}(M)$ acts on $H^{2}(M)$, it preserves the square, the divisibility and the type. Therefore, $\operatorname{Diff}(M)$ acts on $\mathcal{S P \mathcal { H }} \mathcal{H}_{s, p}^{o}(M)$ and $\mathcal{S P} \mathcal{H}_{s, p}^{c}(M)$ separately. Remarkably this action is transitive if $s \geq-1$.

THEOREM D. Let $M$ be a smooth, closed oriented 4-manifold with $\mathcal{C}_{M}$ nonempty. Then $\operatorname{Diff}(M)$ acts transitively on $\mathcal{S P \mathcal { H }} \mathcal{H}_{s, p}^{o}(M)$ and $\mathcal{S P} \mathcal{H}_{s, p}^{c}$ when $s \geq-1$.

The difficult case in Theorem $\mathrm{D}$ is when $M$ is a rational manifold and $s \geq 0$. The proof in this case relies crucially on a new characterization of the action of $\operatorname{Diff}(M)$ on $H^{2}(M ; \mathbf{Z})$ in terms of the $K$-symplectic cones.

We do not know whether the transitivity continues to hold when $s$ is less than -1 . The case $s=-2$ is particularly interesting and will be the subject of further investigation. We remark that Theorem $\mathrm{D}$ will be applied in [L2] to prove that the fiber sums of relatively minimal Lefschetz fibrations are minimal manifolds.

Convention. From now on, when we say an integral cohomology class is represented by a surface, we mean its Poincaré dual is represented by a surface.

The organization of the paper is as follows. In $\S 2$, we study which automorphisms of the cohomology lattice of a rational manifold are realized by diffeomorphisms. Based on a characterization by Friedman and Morgan in [FM1-2], we give a new characterization in terms of the $K$-symplectic cones. This new characterization will be used in $\S 4$ and is one major new theoretical input in this paper. In $\S 3$, we systematically study the symplectic genus and prove Theorem A and B. Most of this section is a series of computational lemmas which give enough case-by-case control to prove the theorems. In $\S 4$, we study the problem of representing a class by spheres and determine the action of diffeomorphism groups on $\mathcal{S P H}(M)$. Theorems $\mathrm{C}$ and $\mathrm{D}$ will be proved there.

The authors would like to thank Janos Kollár, Ronnie Lee, Robert Friedman and Gang Tian for helpful discussions. This research is partially supported by NSF.

2. Diffeomorphism group of rational and $K$-symplectic cones. On a manifold $M$, each diffeomorphism induces an automorphism of the lattice of the second integral cohomology. Hence there is a natural map from $\operatorname{Diff}(M)$ to the automorphism group of the lattice. Let $\mathrm{D}(M)$ be the image of this natural map. In other words, an automorphism is in $\mathrm{D}(M)$ if it is realized by an orientation-preserving diffeomorphism. We will describe $\mathrm{D}(M)$ for both rational and irrational ruled manifolds.

On each irrational ruled manifold $M$, there is a class (unique up to sign) with square zero whose Poincáre dual is represented by an embedded sphere. It is proved in [FM2] that an automorphism of the cohomology lattice is in $\mathrm{D}(M)$ if and only if that class is preserved up to sign. In particular, the -Id automorphism is in $\mathrm{D}(M)$.

The case for rational manifolds is rather complicated. Each rational manifold $M$ is of the form $C P^{2} \# n \overline{C P}^{2}$. When $n \leq 9$, a result of Wall states that any automorphism is realized by a diffeomorphism. The more difficult case $n \geq 10$ requires the concepts of $P$-cell and super $P$-cell introduced by Friedman and Morgan [FM1], and a characterization of the diffeomorphism group via these terms. In fact they are 
not adequate for the purpose of this paper, and we need to consider their partial compactifications and relate them to the $K$-symplectic cones.

Suppose $M$ is an oriented closed manifold with $b^{+}=1, b^{-}=n$ and no torsion in $H^{2}(M ; \mathbf{Z})$. A basis $\left(x, \alpha_{1}, \ldots, \alpha_{n}\right)$ for $H^{2}(M ; \mathbf{Z})$ is called standard if $x^{2}=1$, and $\alpha_{i}^{2}=-1$ for each $i=1, \ldots, n$. Let

$$
\begin{aligned}
\mathcal{P} & =\left\{e \in H^{2}(M ; \mathbf{R}) \mid e \cdot e>0\right\} \\
\mathcal{B} & =\left\{e \in H^{2}(M ; \mathbf{R}) \mid e \cdot e=0\right\} \\
\overline{\mathcal{P}} & =\left\{e \in H^{2}(M ; \mathbf{R}) \mid e \cdot e \geq 0\right\}
\end{aligned}
$$

For each class $x \in H^{2}(M ; \mathbf{Z})$ with $x^{2}<0$, we define $x^{\perp} \in H^{2}(M ; \mathbf{R})$ to be the orthogonal subspace to $x$ with respect to the cup product, and we call $\left(x^{\perp}\right) \cap \mathcal{P}$ the wall in $\mathcal{P}$ defined by $x$. Let $\mathcal{W}_{1}$ be the set of walls in $\mathcal{P}$ defined by integral classes with square -1 . A chamber for $\mathcal{W}_{1}$ is the closure in $\mathcal{P}$ of a connected component of $\mathcal{P}-\cup_{W \in \mathcal{W}_{1}} W$.

Any point $x \in \mathcal{P}$ with square 1 at which $n$ mutually perpendicular walls of $\mathcal{W}_{1}$ meet is called a corner. Any corner is an integral class (see Lemma 2.2 in [FM1]). Suppose $C$ is a chamber for $\mathcal{W}_{1}$. If $x$ is a corner in $C$, a standard basis $\left(x, \alpha_{1}, \ldots, \alpha_{n}\right)$ for $H^{2}(M ; \mathbf{Z})$ is called a standard basis adapted to $C$ if $\alpha_{i} \cdot C \geq 0$ for each $i$. The canonical class of the pair $(x, C)$ is defined to be $\kappa(x, C)=3 x-\sum_{i} \alpha_{i}$. Suppose $C$ is a chamber for $\mathcal{W}_{1}$ and $x$ is a corner in $C$, we define

$$
P(x, C)=C \cap\{e \in \mathcal{P} \mid \kappa(x, C) \cdot e \geq 0\} .
$$

Any subset of $\mathcal{P}$ of the form $P(x, C)$ is called a $P$-cell.

Notation. For any $U \subset \mathcal{P}$ (similarly $\mathcal{B}, \overline{\mathcal{P}}$ ), we will use $\operatorname{int}_{\mathcal{P}}(U)$ (similarly $\left.\operatorname{int}_{\mathcal{B}}(U), \operatorname{int}_{\overline{\mathcal{P}}}(U)\right)$ to denote $U \cap \operatorname{int}(\mathcal{P})(\operatorname{similarly} U \operatorname{int}(\mathcal{B}), U \cap \operatorname{int}(\overline{\mathcal{P}}))$. For any $V \subset$ $\overline{\mathcal{P}}$ (respectively $\mathcal{P}$ ), we will use $\bar{V}$ (similarly $\hat{V}$ ) to denote its closure in $\overline{\mathcal{P}}$ (similarly $\mathcal{P})$.

The basic properties of $P$-cells are summarized in the following lemma.

LEMMA 2.1.

1. A $P$-cell is a chamber for the set of walls $\mathcal{W}_{1} \cup\left\{\kappa(x, C)^{\perp} \cap \mathcal{P}\right\}$.

2. If $P(x, C)=P\left(x^{\prime}, C^{\prime}\right)$, then $\kappa(x, C)=\kappa\left(x^{\prime}, C^{\prime}\right)$. Thus for each $P$-cell $P$ we can assign a unique canonical class of the form $\kappa(x, C)$, which will be written as $\kappa(P)$.

3. If $\psi$ is an automorphism of the lattice and $P$ is a $P$-cell with canonical class $\kappa$, then $\psi \cdot P$ is also a $P$-cell with canonical class $\psi(\kappa)$.

4. If $P$ and $P^{\prime}$ are distinct $P$-cells, then $\operatorname{int}_{\mathcal{P}}(P)$ and $\operatorname{int}_{\mathcal{P}}\left(P^{\prime}\right)$ are disjoint.

5. If $P$ and $P^{\prime}$ are distinct $P$-cells, then $\operatorname{int}_{\mathcal{B}}(\bar{P} \cap \mathcal{B})$ and $\operatorname{int}_{\mathcal{B}}\left(\bar{P}^{\prime} \cap \mathcal{B}\right)$ are disjoint. In other words, the interiors of the $\mathcal{B}$-boundaries of the closure of distinct $P$-cells are also disjoint.

Proof. The proofs of the first 4 properties can be found in chapter II in [FM 1]. Here we prove property 5 . Notice that $\overline{\mathcal{P}}=\mathcal{P} \cup \mathcal{B}$. If $x$ is any point in $\operatorname{int}_{\mathcal{B}}(\bar{P} \cap \mathcal{B})$, then the intersection of $\mathcal{P}$ with any sufficiently small neighborhood in $\overline{\mathcal{P}}$ of $x$ is nonempty and is contained in $\operatorname{int}_{\mathcal{P}}(P)$. Thus if $\operatorname{int}_{\mathcal{B}}(\bar{P} \cap \mathcal{B})$ and $\operatorname{int}_{\mathcal{B}}\left(\bar{P}^{\prime} \cap \mathcal{B}\right)$ intersect, then $\operatorname{int}_{\mathcal{P}}(P)$ and $\operatorname{int}_{\mathcal{P}}\left(P^{\prime}\right)$ overlap and hence they are the same $P$-cells by property 4. Therefore distinct $P$-cells have disjoint $\mathcal{B}$-boundaries.

It turns out that $P$-cells are closely related to the $K$-symplectic cone introduced in [LLiu1]. Let us recall the definition of $K$-symplectic cone. A class $K \in H^{2}(M ; \mathbf{Z})$ 
is called a symplectic canonical class if it is the canonical class of some orientationcompatible symplectic structures. Let $\mathcal{K}$ be the set of symplectic canonical classes. For any $K \in \mathcal{K}$ we introduce the $K$-symplectic cone:

$$
\mathcal{C}_{K}=\left\{e \in \mathcal{C}_{M} \mid e=[\omega] \text { for some } \omega \in \Omega_{K}\right\}
$$

where $\Omega_{K}$ is the set of orientation-compatible symplectic forms with $K$ as the symplectic canonical class. It is shown in [LLiu2] that $\mathcal{C}_{K}$ is disjoint from $\mathcal{C}_{K^{\prime}}$ if $K \neq K^{\prime}$. For a manifold with $b^{+}=1$ and any $K \in \mathcal{K}$, we can in fact determine $\mathcal{C}_{K}$ in terms of a certain subset of $\mathcal{E}_{M}$. Recall that $\mathcal{E}_{M}$ is the set of integral cohomology classes represented by smoothly embedded spheres of square -1 . When there is no confusion we will omit the subscript $M$. Introduce the set of $K$-exceptional spheres as

$$
\mathcal{E}_{K}=\{E \in \mathcal{E} \mid K \cdot E=-1\}
$$

It is proved in Theorem 4 in [LLiu1] that

$$
\mathcal{C}_{K}=\left\{e \in \mathcal{P} \mid e \cdot E>0 \text { for any } E \in \mathcal{E}_{K}\right\}
$$

Let $\hat{\mathcal{C}}_{K}$ be the closure of $\mathcal{C}_{K}$ in $\mathcal{P}$. Then it is not hard to prove

$$
\hat{\mathcal{C}}_{K}=\left\{e \in \mathcal{P} \mid e \cdot E \geq 0 \text { for any } E \in \mathcal{E}_{K}\right\}
$$

In order to link the $P$-cells and the symplectic cones, we also need to consider good generic surfaces as in [FM1]. A good generic surface $X$ is an algebraic surface such that the anti-canonical divisor is effective and smooth, and that any smooth rational curve has square no less than -1 . All such surfaces are rational surfaces and can be holomorphically blown down to $C P^{2}$ (see I.2 in [FM1]). Let $\rho: X \longrightarrow C P^{2}$ be a holomorphic blow down with exceptional fibers $F_{1}, \ldots, F_{n}$, where each $F_{i}$ is an embedded rational curve with square -1 . Let $K_{X}$ be the canonical class of $X$. Then $-K_{X}=3 \rho^{*}(H)-\sum_{i=1}^{n} F_{i}$, where $H$ is a hyperplane section of $C P^{2}$.

The surface $X$ has many Kähler metrics. Associated with each such metric is its Kähler form and associated cohomology class in $H^{2}(X ; \mathbf{R})$. The Kähler cone $\mathcal{A}(X)$ of $X$ is then the set of all Kähler cohomology classes. By the Nakai-Moishezon criterion, the Kähler cone $\mathcal{A}(X)$ consists of all the classes in $\mathcal{P}$ which pair positively on any holomorphic curve. Let $\hat{\mathcal{A}}(X)$ be the closure of $\mathcal{A}(X)$ in $\mathcal{P}$.

Proposition 2.2. Let $X$ be a good generic surface. Let $P_{0}$ be the $P$-cell containing the class $\rho^{*} H$. Then $P_{0}$ coincides with $\hat{\mathcal{A}}(X)$, and $\kappa\left(P_{0}\right)=-K_{X}$. Moreover,

$$
P_{0}=\left\{e \in \hat{\mathcal{C}}_{K_{X}} \mid e \cdot\left(-K_{X}\right) \geq 0\right\}
$$

Proof. The first statement is proved in II. 3 and II.4 in [FM1]. So we only need to show that $\hat{\mathcal{A}}(X)$ consists of all classes in $\hat{\mathcal{C}}_{K_{X}}$ which pair non-negatively with $\left(-K_{X}\right)$.

Since a Kähler form is a symplectic form, the Kähler cone $\mathcal{A}(X)$ is certainly a subset of the $K_{X}$-symplectic cone $\mathcal{C}_{K_{X}}$. Therefore $\hat{\mathcal{A}}(X)$ is a subset of $\hat{\mathcal{C}}_{K_{X}}$. To prove the inclusion in the other direction, we need the following result:

$$
\hat{\mathcal{A}}(X)=\left\{e \in \mathcal{P} \mid e \cdot(-K) \geq 0 \text { and } e \cdot E \geq 0 \text { for any } E \in \mathcal{E}^{h o l}(X)\right\}
$$


which is Proposition 3.4 in [FM1]. Here $\mathcal{E}^{\text {hol }}(X)$ is the set of embedded rational curves with square -1 . With this characterization of $\hat{\mathcal{A}}(X)$ we just have to show that, on a good generic surface, any class $e \in \hat{\mathcal{C}}_{K_{X}}$ with $e \cdot\left(-K_{X}\right) \geq 0$ is non-negative on any class in $\mathcal{E}^{\text {hol }}(X)$. This follows from the obvious inclusion $\mathcal{E}^{\text {hol }}(X) \subset \mathcal{E}_{K_{X}}$. The proof is complete.

REMARK 2.3. From Proposition 2.2, we find

$$
P_{0}=\left\{e \in \mathcal{P} \mid e \cdot\left(-K_{X}\right) \geq 0 \text { and } e \cdot E \geq 0 \text { for any } E \in \mathcal{E}^{\text {hol }}(X)\right\} .
$$

Since $P_{0}$ coincides with $\hat{A}(X)$, it is possible that the two sets $\mathcal{E}^{\text {hol }}(X)$ and $\mathcal{E}_{K_{X}}$ are the same.

LEMMA 2.4. Let $M$ be a rational 4-manifold. For each $K \in \mathcal{K}$, there exists a $P$-cell $P_{K}$ such that $\kappa\left(P_{K}\right)=-K$ and

$$
P_{K}=\left\{e \in \hat{\mathcal{C}}_{K} \mid e \cdot(-K) \geq 0\right\}
$$

Proof. Suppose $X$ is a good generic surface and $M$ is the underlying rational 4-manifold. By the result in [LLiu1] that $\operatorname{Diff}(M)$ acts transitively on $\mathcal{K}$, there is a diffeomorphism $\phi$ of ' $M$ such that $\phi^{*}\left(K_{X}\right)=K$. Since $\phi^{*} \omega \in \Omega_{\phi^{*}\left(K_{X}\right)}$ for any $\omega \in \Omega_{K_{X}}$, we have $\mathcal{C}_{K}=\phi^{*} \mathcal{C}_{K_{X}}$. Thus by Proposition 2.2 we have

$$
\phi^{*}\left(P_{0}\right)=\left\{e \in \hat{\mathcal{C}}_{K} \mid e \cdot(-K) \geq 0\right\}
$$

Let $P_{K}=\phi^{*} P_{0}$. By Lemma 2.1(2-3), $P_{K}$ is still a $P$-cell with canonical class $K$. The proof is complete.

Now we introduce super $P$-cell, which is defined via a reflection group associated to a $P$-cell. Suppose $\gamma$ is a class with square -1 or -2 . We can define an automorphism of the lattice as follows,

$$
R(\gamma) \beta=\beta+\frac{2(\gamma \cdot \beta)}{|\gamma \cdot \gamma|} \gamma
$$

This automorphism $R(\gamma)$ is called the reflection along $\gamma$. For a $P$-cell $P$ define $\mathcal{G}_{P}$ to be the set

$$
\left\{\alpha \mid \alpha^{2}=-1, \alpha \neq \kappa(P) \text { and } \alpha \text { defines a wall of } \mathcal{P}\right\} .
$$

Let $\mathcal{R}(P)$ be the group generated by reflections along classes in $\mathcal{G}_{P}$.

The super $P$-cell of $P$ is defined as

$$
S(P)=\cup_{\psi \in \mathcal{R}(P)} \psi(P) .
$$

We will need the following simple fact on reflections.

Lemma 2.5. Suppose $F=\psi\left(F_{0}\right)$ for some $F_{0} \in \mathcal{G}_{P}$. Then $R(F)=\psi \circ R\left(F_{0}\right) \circ \psi^{-1}$. In particular, $R(F) \in \mathcal{R}(P)$.

Proof. For any class $x$, we have

$$
\begin{gathered}
R(F) \circ \psi(x)=\psi(x)+2(F \cdot \psi(x)) F=\psi(x)+2\left(\psi\left(F_{0}\right) \cdot \psi(x)\right) \psi\left(F_{0}\right) \\
\psi \circ R\left(F_{0}\right)(x)=\psi\left(x+2\left(F_{0} \cdot x\right) F_{0}\right)=\psi(x)+2\left(\psi\left(F_{0}\right) \cdot \psi(x) \psi\left(F_{0}\right) .\right.
\end{gathered}
$$


So $R(F) \circ \psi=\psi \circ R\left(F_{0}\right)$, and the statements follow.

Proposition 2.6. Let $M$ be a rational 4-manifold. Any good generic surface $X$ with $M$ as the underlying manifold gives rise to a $P$-cell of $M$, denoted by $P_{0}$.

1. If $\phi$ is an automorphism, then $\phi(S(P))$ is also a super $P$-cell. In particular, $-S(P)$ is a super $P$-cell.

2. An automorphism is in $\mathrm{D}(M)$ if and only if it preserves the distinguished super $P$-cell $S\left(P_{0}\right)$ up to sign. Consequently, $\mathrm{D}(M)$ is generated by $-\mathrm{Id}, \mathcal{R}\left(P_{0}\right)$ and the isotropy subgroup of $P_{0}$.

3. If $\operatorname{int}_{\mathcal{P}}(S(P)) \cap \operatorname{int}_{\mathcal{P}}\left(S\left(P^{\prime}\right)\right)$ is not empty, then $S(P)=S\left(P^{\prime}\right)$.

4. If $\operatorname{int}_{\mathcal{B}}(\overline{S(P)} \cap \mathcal{B})$ and $\operatorname{int}_{\mathcal{B}}\left(\overline{S\left(P^{\prime}\right)} \cap \mathcal{B}\right)$ intersect, then $S(P)=S\left(P^{\prime}\right)$.

5. If $\operatorname{int}_{\overline{\mathcal{P}}}(\overline{\phi(S(P))})$ and $\operatorname{int}_{\overline{\mathcal{P}}}\left(\overline{\phi\left(S\left(P^{\prime}\right)\right)}\right)$ intersect, then $S(P)=S\left(P^{\prime}\right)$.

Proof. The first three properties are in chapter II in [FM1]. The proof of property 4 goes exactly along the line of the proof of the analogous property for the $P$-cells in Lemma 2.1. If $x$ is any point in $\operatorname{int}_{\mathcal{B}}(\overline{S(P)} \cap \mathcal{B})$, then the intersection of $\mathcal{P}$ with any sufficiently small neighborhood in $\overline{\mathcal{P}}$ of $x$ is non-empty and is contained in $\operatorname{int}_{\mathcal{P}}(S(P))$. Thus if $\operatorname{int}_{\mathcal{B}}(\overline{S(P)} \cap \mathcal{B})$ and $\operatorname{int}_{\mathcal{B}}\left(\overline{S\left(P^{\prime}\right)} \cap \mathcal{B}\right)$ intersect, then $S(P)$ and $S\left(P^{\prime}\right)$ have overlapping interiors and hence they are the same super $P$-cells by property 3 .

Notice that

$$
\operatorname{int}_{\overline{\mathcal{P}}}(\overline{\phi(S(P))})=\operatorname{int}_{\mathcal{P}}(S(P)) \cup \operatorname{int}_{\mathcal{B}}(\overline{S(P)} \cap \mathcal{B}) .
$$

The last statement follows immediately from the properties 3 and 4 . The proof is complete.

In the next proposition we are going to relate the super $P$-cells $\pm S\left(P_{0}\right)$ to the $K$-symplectic cones.

Proposition 2.7. Define $M$ and $X$ as in Proposition 2.5. Let $K_{0}$ be the canonical class of $P_{0}$. Then every $K \in \mathcal{K}$ is of the form $\pm \psi\left(K_{0}\right)$, where $\psi \in \mathcal{R}\left(P_{0}\right)$. Consequently,

$$
\begin{aligned}
S\left(P_{0}\right) \cup-S\left(P_{0}\right) & =\cup_{K \in \mathcal{K}} P_{K} \\
\overline{S\left(P_{0}\right) \cup-S\left(P_{0}\right)} \cap \mathcal{B} & =\cup_{K \in \mathcal{K}} \bar{P}_{K} \cap \mathcal{B} .
\end{aligned}
$$

Proof. This is a consequence of the result in [LLiu1] which states that $\mathrm{D}(M)$ acts transitively on $\mathcal{K}$. The positive cone $\mathcal{P}$ has two connected components. Let $K$ be a symplectic canonical class such that $\mathcal{C}_{K}$ and $\mathcal{C}_{K_{0}}$ are in the same connected component of $\mathcal{P}$. Since $\mathrm{D}(M)$ acts transitively on $\mathcal{K}$, there exists $\psi^{\prime} \in \mathrm{D}(M)$ such that $\psi\left(K_{0}\right)=K$. By Proposition 2.6(2), $\psi^{\prime}\left(S\left(P_{0}\right)\right)= \pm S\left(P_{0}\right)$. Since $\psi^{\prime}\left(P_{0}\right)$ is still in the same component of $P_{0}, \psi^{\prime}\left(S\left(P_{0}\right)\right)=S\left(P_{0}\right)$. Therefore $\psi^{\prime}\left(P_{0}\right)$ is a $P$-cell within $S\left(P_{0}\right)$. By the definition of a super $P$-cell, $\psi^{\prime}\left(P_{0}\right)=\psi\left(P_{0}\right)$ for some $\psi \in \mathcal{R}(P)$. Therefore $K=\psi\left(K_{0}\right)$. By Lemma 2.1, $\psi\left(P_{K_{0}}\right)$ and $P_{K}$ have the same canonical class and therefore they are identical. Notice we have shown that

$$
\cup_{K \in \mathcal{K}} P_{K} \subset S\left(P_{0}\right) \cup-S\left(P_{0}\right) \text {. }
$$

To prove the inclusion in the other direction, we just need to show that, for any $\psi \in \mathcal{R}(P), \psi\left(P_{0}\right)=P_{K}$ for some $K \in \mathcal{K}$. This is obvious since $K=\psi\left(K_{0}\right)$ is certainly a symplectic canonical class. The proof is finished.

It is mentioned in [FM2] that super $P$-cells are chambers for the walls given by primitive characteristic classes with square $9-n$. We can in fact show that the set of walls for $S\left(P_{0}\right)$ and $-S\left(P_{0}\right)$ is just the set of the symplectic canonical classes. 
Now we are able to present the main result of this section, a characterization of $\mathrm{D}(M)$ in terms of $K$-symplectic cones.

Theorem 2.8. Let $M$ be a rational 4-manifold. An automorphism $\phi$ is in $\mathrm{D}(M)$ if and only if there are $K$ and $K^{\prime}$ in $\mathcal{K}$ and

1. either there are classes $e \in \hat{\mathcal{C}}_{K}$ and $e^{\prime} \in \hat{\mathcal{C}}_{K^{\prime}}$ with $e \cdot(-K)>0$ and $e^{\prime} \cdot\left(-K^{\prime}\right)>0$, such that $e$ is mapped to $e^{\prime}$ by $\phi$,

2. or there are classes $e \in \overline{\mathcal{C}}_{K} \cap \mathcal{B}$ and $e^{\prime} \in \overline{\mathcal{C}}_{K^{\prime}} \cap \mathcal{B}$ with $e \cdot(-K)>0$ and $e^{\prime} \cdot\left(-K^{\prime}\right)>0$, such that $e$ is mapped to $e^{\prime}$ by $\phi$.

Proof. Due to Proposition 2.7, in the first case, we just have to show that $e$ and $e^{\prime}$ are in the interior of $S\left(P_{0}\right) \cup-S\left(P_{0}\right)$. The arguments for $e$ and $e^{\prime}$ are exactly the same, so we will only argue for $e$. By Lemma 2.4,e $\in P_{K}$. If $e$ is in the interior of $P_{K}$, then $e$ is in the interior of $S\left(P_{0}\right) \cup-S\left(P_{0}\right)$ by Proposition 2.7. $e$ may fail to be in the interior of $P_{K}$ only when $e \cdot E=0$ for some $E \in \mathcal{E}_{K}$. Suppose $P_{K}= \pm \psi\left(P_{0}\right)$ for some $\psi \in \mathcal{R}\left(P_{0}\right)$, then $E=\psi\left(E_{0}\right)$ for some $E_{0} \in \mathcal{G}_{P_{0}}$. By Lemma 2.5, the $P$-cell obtained by reflecting $P_{K}$ along $E$ is still in $S\left(P_{0}\right) \cup-S\left(P_{0}\right)$. Thus we see that $e$ must be in the interior of $S\left(P_{0}\right) \cup-S\left(P_{0}\right)$.

The proof in the second case is similar. We just have to show that $e$ and $e^{\prime}$ are in $\operatorname{int}_{\mathcal{B}}\left(\overline{S\left(P_{0}\right) \cup-S\left(P_{0}\right)} \cap \mathcal{B}\right)$ and we only have to argue for $e$. By Lemma 2.4, $e \in \bar{P}_{K} \cap \mathcal{B}$. $e$ may fail to be in the interior of $\bar{P}_{K} \cap \mathcal{B}$ only when $e \cdot E=0$ for some $E \in \mathcal{E}_{K}$. However by Lemma 2.5 , the reflection of $\bar{P}_{K} \cap \mathcal{B}$ along $E$ is still in $\overline{S\left(P_{0}\right)} \cap \mathcal{B}$. Thus we see that $e$ must be in the interior of $\overline{S\left(P_{0}\right) \cup-S\left(P_{0}\right)} \cap \mathcal{B}$. The theorem is proved.

3. Symplectic genus. We first give the formal definition of the symplectic genus for manifolds with non-empty symplectic cone. For any integral class $e \in \mathcal{P}$, we first define a subset of $\mathcal{K}$,

$$
\mathcal{K}_{e}=\left\{K \in \mathcal{K} \mid \text { there exists a class } \tau \in \mathcal{C}_{K} \text { such that } \tau \cdot e>0\right\}
$$

We further define a subset of $\mathcal{K}_{e}$,

$$
\mathcal{K}(e)=\left\{K \in \mathcal{K}_{e} \mid K \cdot e \geq K^{\prime} \cdot e \text { for any } K^{\prime} \in \mathcal{K}_{e}\right\}
$$

Definition 3.1. Let $K$ be any class in $\mathcal{K}(e)$. The symplectic genus of $e$ is defined to be

$$
\eta(e)=\frac{1}{2}\left(e \cdot K+e^{2}\right)+1
$$

We now list some simple properties of symplectic genus.

LEMMA 3.2.

1. The symplectic genus is no bigger than the minimal genus. Furthermore, if a class is represented by a connected symplectic surface, then its symplectic genus is equal to its minimal genus.

2. $\eta(-e)=\eta(e)$.

3. For any positive integer $p$,

$$
\eta(p e)=p \eta(e)-(p-1)+\frac{\left(p^{2}-p\right)}{2} e \cdot e .
$$

In particular, $\eta(p e) \neq 0$ when $e \cdot e=0$ and $p \geq 2$. 
4. The symplectic genus is invariant under the action of the group of orientationpreserving diffeomorphisms.

5. The symplectic genus of any class of a sufficiently large multiple of any class of positive square is positive.

Proof. Property 1 is a consequence of the adjunction inequalities. When $b^{+}>1$ the adjunction inequality in $[\mathrm{KM}],[\mathrm{MST}],[\mathrm{OS}]$ and [T2] asserts that the genus $g$ of any embedded surface representing $e$ satisfies

$$
2 g-2 \geq|K \cdot e|+e \cdot e
$$

for any symplectic canonical class $K$.

When $b^{+}=1$ and $e$ has non-negative square, the adjunction inequality in [LLiu2] asserts that

$$
2 g-2 \geq K \cdot e+e \cdot e
$$

for any symplectic canonical class $K \in \mathcal{K}_{e}$.

When $e$ has negative square, inequality (3.2) still holds and is basically proved in $\S 3$ in [OS]. We explain here briefly. Suppose $\omega$ is a symplectic form whose class $\tau$ pairs positively with $e$, and let $K(\omega)$ be its symplectical canonical class. Let $s_{0}$ be the canonical Spin ${ }^{c}$ structure with $c_{1}\left(s_{0}\right)=-K(\omega)$. The class $e$ determines another $\operatorname{Spin}^{c}$ structure, denoted by $s_{0}-e$. Suppose $e$ is represented by an embedded surface of genus $h$ such that

$$
2 h-2<K(\omega) \cdot e+e \cdot e .
$$

Then, by Theorem 1.3 in [OS] and the corresponding result in [FS], in a common chamber for both $s_{0}$ and $s_{0}-e$ which is perpendicular to $e$, the Seiberg-Witten invariant of $s_{0}$ being nontrivial implies that the invariant of $s_{0}-e$ is nontrivial as well. The $\omega$-symplectic chamber is such a chamber. Moreover, according to Taubes ([T1]), in this chamber, the Seiberg-Witten invariant of $s_{0}$ is nontrivial. Therefore, in the $\omega$-symplectic chamber, the Seiberg-Witten invariant of $s_{0}-e$ is nontrivial as well. By another result of Taubes ([T2]), $\tau \cdot(-e)>0$. This contradicts our assumption, so inequality (3.2) still holds in this case. Therefore, in any case, we have $m(e) \geq \eta(e)$.

Suppose that $e$ is represented by a genus $h$ symplectic surface with respect to a symplectic form $\omega$. Then $\omega$ is positive on this surface. If $K(\omega)$ is the symplectic canonical class of $\omega$, then $K(\omega) \in \mathcal{K}_{e}$ and $2 h-2=K(\omega) \cdot e+e \cdot e$. Together with inequalities (3.1) and (3.2), we see that $h=m(e)=\eta(e)$.

If $K$ is the symplectic canonical class of a symplectic form $\omega$, then $-K$ is the symplectic canonical class of the symplectic form $-\omega$. Therefore,

$$
\mathcal{K}_{-e}=\left\{-K \mid K \in \mathcal{K}_{e}\right\} \text { and } \mathcal{K}(-e)=\{-K \mid K \in \mathcal{K}(e)\}
$$

And $\eta(-e)=\eta(e)$ is an immediate consequence of equation (3.3).

For any positive integer $p$, we have

$$
\mathcal{K}_{e}=\mathcal{K}_{p e} \text { and } \mathcal{K}(e)=\mathcal{K}(p e)
$$

The formula for $\eta(e)$ then follows from equation (3.4) with a straightforward calculation. When $e \cdot e=0, \eta(p e)$ is therefore given by $p(\eta(e)-1)+1$. Evidently it is not divisible by $p$ and concequently cannot be zero if $p \geq 2$. 
It is shown in Proposition 4.1 in [LLiu1] that, if $\phi$ is an orientation-preserving diffeomorphism, then $\phi^{*} \mathcal{C}_{K}=\mathcal{C}_{\phi^{*} K}$. It follows that

$$
\phi^{*} \mathcal{K}_{e}=\mathcal{K}_{\phi^{*} e} \text { and } \phi^{*} \mathcal{K}(e)=\mathcal{K}\left(\phi^{*} e\right)
$$

Property 4, then, is an immediate consequence of equation (3.5).

The last property follows directly from the definition. Let $e$ be a class with positive square. When $N$ is large, $N e \cdot N e$ dominates $N e \cdot K$ for any $K \in \mathcal{K}(e)$, and therefore $N e$ has positive symplectic genus. Lemma 3.2 is proved.

Now we set out to prove Theorem $B$. The proof requires the notion of reduced classes for non-minimal rational and irrational ruled manifolds (for rational manifolds, it is introduced in [Ki] and [G]). A nice property of this notion is that every class with positive square can be transformed in an explicit way to a reduced class via diffeomorphisms. Thus by Lemma 3.2(4) we only have to show that Theorem B holds for any reduced class $e$.

To introduce the reduced class let us review the minimal reductions of a rational or irrational ruled manifold. The only minimal rational manifolds are $C P^{2}$ and $S^{2} \times S^{2}$. And a non-minimal rational manifold has two kinds of decomposition- it is either decomposed as $C P^{2} \# n \overline{C P}^{2}$ or as $S^{2} \times S^{2} \#(n-1) \overline{C P}^{2}$. We will always use the first decomposition and call it a standard decomposition. The picture for irrational ruled manifolds is similar. $S^{2}$-bundles over a Riemann surface of positive genus are the only minimal irrational ruled manifolds. Fix the base surface $\Sigma_{g}$, there are two $S^{2}$-bundles over it, the trivial one $S^{2} \times \Sigma_{g}$ and the unique non-trivial one $S^{2} \tilde{\times} \Sigma_{g}$. A non-minimal irrational ruled manifold also has two types of decomposition, either as $S^{2} \times \Sigma_{g} \# n \overline{C P}^{2}$ or as $S^{2} \tilde{\times} \Sigma_{g} \# n \overline{C P}^{2}$. We will use the first decomposition and call it a standard decomposition.

Let $H$ be a generator of $H^{2}\left(C P^{2} ; \mathbf{Z}\right)$ and $E_{1}, \ldots, E_{n}$ be the generators of the $\overline{C P}^{2}$. Let $U$ and $T$ be classes in $S^{2} \times \Sigma_{g}$ represented by $\{p t\} \times \Sigma_{g}$ and $S^{2} \times\{p t\}$ respectively. $H, E_{1}, \ldots, E_{n}$ are naturally considered as classes in $H^{2}\left(C P^{2} \# n \overline{C P}^{2} ; \mathbf{Z}\right)$ and form a basis. We will call such a basis a standard basis. Similarly, $U, T, E_{1}, \ldots, E_{n}$ are naturally considered as classes in $H^{2}\left(S^{2} \times \Sigma_{g} \# n \overline{C P}^{2} ; \mathbf{Z}\right)$ and form a basis. Such a basis is also called a standard basis. Given such a basis, according to Wall ([W]), an automorphism is called trivial if either it permutes the $E_{i}$ or it is a reflection along an $E_{i}$. It was shown in [W] that trivial automorphisms are in $\mathrm{D}(M)$.

On $C P^{2} \# n \overline{C P}^{2}$, let $K_{0}=-3 H+\sum_{i} E_{i}$; and on $S^{2} \times \Sigma_{g} \# n \overline{C P}^{2}$, let $K_{0}=$ $-2 U+(2 g-2) T+\sum_{i} E_{i}$. By the blow up construction (see e.g. [Mc1]) $K_{0}$ is a symplectic canonical class.

DEFinition 3.3. For a non-minimal rational manifold with a standard decomposition $C P^{2} \# n \overline{C P}^{2}$ and a standard basis $\left\{H, E_{1}, \ldots, E_{n}\right\}$, a class $\xi=a H-\sum_{i=1}^{n} b_{i} E_{i}$ is called reduced if

$$
\left\{\begin{array}{l}
b_{1} \geq b_{2} \geq \cdots \geq b_{n} \geq 0 \\
a \geq b_{1}+b_{2}+b_{3} .
\end{array}\right.
$$

For a non-minimal irrational ruled manifold with a standard decomposition $S^{2} \times$ $\Sigma_{g} \# n \overline{C P}^{2}$ and a standard basis $\left\{U, T, E_{1}, \ldots, E_{n}\right\}$, a class $e=a U+b T-\sum c_{i} E_{i}$ is called reduced if

$$
\left\{\begin{array}{l}
a \geq 0, c_{1} \geq c_{2} \geq \cdots \geq c_{n} \geq 0 \\
a \geq c_{i} \text { for any } i .
\end{array}\right.
$$


Reduced classes have the following properties:

LEMMA 3.4. Let $M$ be a non-minimal rational or irrational ruled manifold with a standard decomposition and a standard basis.

1. Any class of non-negative square is equivalent to a reduced class under the action of orientation-preserving diffeomorphisms. Moreover we can find such a diffeomorphism by a simple algorithm.

2. For a class with square -1 , when $b^{-}(M) \neq 2$, it either has reduced form or is equivalent to the class $E_{1}$; when $b^{-}(M)=2$, another possibility is that it is characteristic, and equivalent to $H-E_{1}-E_{2}$ in the rational case and to $T-E_{1}$ in the ruled case. Similarly, for a class with square -2 , when $b^{-}(M) \neq 3$, it either has reduced form or is equivalent to the class $E_{1}+E_{2}$; when $b^{-}(M)=3$, another possibility is that it is characteristic, and equivalent to $H-E_{1}-E_{2}-E_{3}$ in the rational case and to $T-E_{1}-E_{2}$ in the irrational ruled case.

3. If $e$ is reduced, then $e \cdot F \geq 0$ for any $F \in \mathcal{E}_{K_{0}}$.

4. If $e$ is reduced, then $K_{0} \in \mathcal{K}_{e}$.

5 . If $e$ is a reduced class with non-negative square, then $K_{0} \in \mathcal{K}(e)$, and consequently $\eta(e)$ is given by $\left(K_{0} \cdot e+e \cdot e\right) / 2+1$.

Proof. We divide the proof into two cases.

(i). First consider a non-minimal rational manifold with a standard decomposition $C P^{2} \# n \overline{C P}^{2}$ and a standard basis. When $n \leq 9$, all the properties have been established (for 1 and 4 see [Li1], for 2 see [LiL2]). So we assume that $n \geq 10$.

Property 1. In fact, it was also proved in [Li1]. Since we will use the similar arguments to prove property 2 , we provide some details here. Suppose $e=a H-$ $\sum_{i} b_{i} E_{i}$ is a class with non-negative square. First of all, by the trivial automorphisms, we can arrange so that $a \geq 0$ and $b_{1} \geq b_{2} \geq \ldots \geq b_{n} \geq 0$. When $n \geq 3$, the class $H-E_{1}-E_{2}-E_{3}$ is represented by an embedded sphere with square -2 . So the reflection along $H-E_{1}-E_{2}-E_{3}$ is an automorphism in $\mathrm{D}(M)$. Under this reflection, $a$ is mapped to $a^{\prime}=2 a-\left(b_{1}+b_{2}+b_{3}\right)$. If $e$ is not already reduced and $2 a-\left(b_{1}+b_{2}+b_{3}\right) \geq 0$, then $0 \leq a^{\prime}<a$. From this we see the process can be repeated either to lead to a reduced class or to a class with $2 \tilde{a}-\left(\tilde{b}_{1}+\tilde{b}_{2}+\tilde{b}_{3}\right)<0$. However, if $2 \tilde{a}<\left(\tilde{b}_{1}+\tilde{b}_{2}+\tilde{b}_{3}\right)$, then from

$$
\tilde{a}^{2} \geq \sum_{i} \tilde{b}_{i}^{2} \quad \text { and } \quad\left(\tilde{b}_{1}+\tilde{b}_{2}+\tilde{b}_{3}\right)^{2} \leq 3\left(\tilde{b}_{1}^{2}+\tilde{b}_{2}^{2}+\tilde{b}_{3}^{2}\right)
$$

we have

$$
\left(\tilde{b}_{1}^{2}+\tilde{b}_{2}^{2}+\tilde{b}_{3}^{2}\right) \leq \tilde{a}^{2}<(3 / 4)\left(\tilde{b}_{1}^{2}+\tilde{b}_{2}^{2}+\tilde{b}_{3}^{2}\right) .
$$

This is an obvious contradiction.

Property 2. Suppose we have a class $e$ of square -1 . The same argument as above proves that $e$ is either equivalent to a reduced class or a class with

$$
\left(\tilde{b}_{1}^{2}+\tilde{b}_{2}^{2}+\tilde{b}_{3}^{2}\right)-1 \leq \tilde{a}^{2}<(3 / 4)\left(\tilde{b}_{1}^{2}+\tilde{b}_{2}^{2}+\tilde{b}_{3}^{2}\right) .
$$

In the latter case $\left(\tilde{b}_{1}^{2}+\tilde{b}_{2}^{2}+\tilde{b}_{3}^{2}\right)<4$ and we easily deduce that, up to trivial automorphisms, the only such class is $E_{1}$. For a class with square -2 , the same argument again proves that $e$ is either equivalent to a reduced class or a class with

$$
\left(\tilde{b}_{1}^{2}+\tilde{b}_{2}^{2}+\tilde{b}_{3}^{2}\right)-2 \leq \tilde{a}^{2}<(3 / 4)\left(\tilde{b}_{1}^{2}+\tilde{b}_{2}^{2}+\tilde{b}_{3}^{2}\right) .
$$


In the latter case we easily find that there are only two possibilities up to trivial automorphisms: $H-E_{1}-E_{2}-E_{3}$ and $E_{1}+E_{2}$. However $H-E_{1}-E_{2}-E_{3}$ is equivalent to $E_{1}+E_{2}$ under the reflection along $H-E_{2}-E_{3}-E_{4}$ and trivial automorphisms.

Property 3. Assume that $F=t H-\sum s_{i} E_{i}$. Then

$$
K_{0} \cdot F=-3 t+\sum s_{i}=-1 \text { and } e \cdot F=a t-\sum s_{i} b_{i}
$$

It was shown in [LLiu2] that $F, E_{1}, \ldots, E_{n}$ and $H$ are all represented by connected smooth $J$-holomorphic spheres for some almost complex structure $J$. By the positivity of intersection of distinct $J$-holomorphic curves, $t \geq 0$, and $s_{i} \geq 0$ unless $F=E_{i}$. If $F=E_{i}$ for some $i$, clearly we have $e \cdot F \geq 0$. If $F \neq E_{i}$, then $t>0$ and $t \geq s_{i} \geq 0$. We can divide the $b_{i}$ into $t$ groups, each consisting of no more than three $b_{i}$. Since $s_{i}$ is no bigger than $t$, the division can be made such that the $b_{i}$ in each group have distinct indices. The condition of $e$ being reduced implies that $a-b_{i}-b_{j}-b_{k} \geq 0$ for any $i, j, k$ which are mutually distinct. The property follows.

Property 4. Notice that for any sufficiently small $\epsilon, \omega_{\epsilon}=H-\sum \epsilon E_{i}$ is a symplectic form with canonical class $K_{0}$. Since $\omega_{\epsilon} \cdot e>0$ for $\epsilon$ small, we have $K_{0} \in \mathcal{K}_{e}$.

Property 5. Since a reduced class $e$ with non-negative square has a positive $H$ term, by the light cone lemma in [LLiu2], the class of a symplectic form is positive on $e$ only when it has a positive $H$ term as well. Therefore, if $K$ is any symplectic canonical class in $\mathcal{K}_{e}$, then it has a negative $H$ term. By Proposition 2.5, any $K \in \mathcal{K}_{e}$ is of the form $\psi\left(K_{0}\right)$ for some $\psi \in \mathcal{R}\left(P_{0}\right)$. We claim that $K \cdot e \leq K_{0} \cdot e$ for any $K$ of the form $K \in \mathcal{K}_{e}$. Once this is established it is clear that $K_{0} \in \mathcal{K}(e)$ and property 4 follows. Now we proceed to prove the above claim. Write $\psi$ as $R^{\left(F_{k}\right)} \circ \ldots \circ R^{\left(F_{1}\right)}\left(K_{0}\right)$ where $F_{i} \in \mathcal{E}_{K_{0}}$. Since $K_{0} \cdot F_{i}=-1$, we have

$$
\begin{aligned}
& R^{\left(F_{1}\right)}\left(K_{0}\right)=K_{0}+2\left(K_{0} \cdot F_{1}\right) F_{1}=K_{0}-2 F_{1} \\
& R^{\left(F_{2}\right)}\left(K_{0}-F_{1}\right)=\left[K_{0}-F_{1}\right]+2\left[\left(K_{0}-F_{1}\right) \cdot F_{2}\right] F_{2}=K_{0}-F_{1}-\left(1+F_{1} \cdot F_{2}\right) F_{2} .
\end{aligned}
$$

This, together with the fact $F_{i} \cdot F_{j} \geq 0$ due to positivity of intersection, we have

$$
K=K_{0}-2 F_{1}-\sum_{i=2}^{k} 2 c_{i} F_{i}, \quad c_{i} \geq 1 .
$$

Now the claim follows from property 3.

(ii). Now consider a non-minimal irrational ruled manifold with a standard decomposition and a standard basis. Suppose $e=a U+b T-\sum_{i} c_{i} E_{i}$ is a class.

Properties 1 and 2. We will prove the first two properties together. As we have mentioned, the -Id automorphism is realized by an orientation-preserving diffeomorphism. Therefore we can assume that $a \geq 0$.

The easier case is when $a=0$. In this case, $e \cdot e=-\sum_{i} c_{i}^{2}$. If $e$ has non-negative square, then $c_{i}=0$ for each $i$ and $e$ is simply the class $b T$, which is certainly reduced. If $e$ has square -1 . then $c_{i}= \pm 1$ for some $i$ and $c_{j}=0$ for any $j \neq i$. Consider the reflection along [b/2]T-E $E_{i}$ which maps $e$ to $E_{i}$ or $T+E_{i}$. When $n \geq 1$, the reflection along $T+E_{1}-E_{2}$ maps $T+E_{i}$ to the class $E_{2}$. If $e$ has square -2 , we have $c_{i}=c_{j}=1$ for some $i \neq j$ and $c_{k}=0$ for any $k$ different from $i$ and $j$. 
When $a$ is strictly positive, we will show that under an orientation-preserving diffeomorphism which is a composition of reflections along classes represented by embedded spheres with square $-1, e$ is equivalent to a class $\tilde{e}=a U+\tilde{b}-\sum_{i} \tilde{c}_{i} E_{i}$ with $a \geq \tilde{c}_{i}$ for each $i$. For any $r_{i} \geq 0$ and $\epsilon_{i}= \pm 1$ to be determined, it is easy to see, via the tube construction, that $\mu_{i}=r_{i} T+\epsilon_{i} E_{i}$ is represented by an embedded sphere with square -1 . Therefore, the reflection along $\mu_{i}$ is realized by an orientation-preserving diffeomorphism. Since $e \cdot \mu_{i}=a r_{i}+\epsilon_{i} c_{i}$, under the reflection,

$$
c_{i} \longrightarrow c_{i}^{\prime}=c_{i}-2 a r_{i} \epsilon_{i}-2 c_{i}=-c_{i}-2 a r_{i} \epsilon_{i}
$$

and $a$ is invariant. We first assume that $a$ is positive. In order for $\left|c_{i}^{\prime}\right| \leq a_{i}$, we find that $r_{i}$ and $\epsilon_{i}$ should satisfy

$$
-\frac{1}{2}-\frac{c}{2 a} \leq r_{i} \epsilon_{i} \leq \frac{1}{2}-\frac{c}{2 a}
$$

Clearly, such a pair $\left(r_{i}, \epsilon_{i}\right)$ exists, and there is a unique solution when $c / a$ is not an odd integer, and there are two solutions when $c /(2 a)$ is an odd integer. By applying this process for each $i$, we obtain a desired class $\tilde{e}$. Notice that $\tilde{e}$ is equivalent to a reduced class under trivial automorphisms. So we have proved that $e$ is equivalent to a reduced class if $a>0$.

Property 3. It is a immediate consequence of the fact (see [Bi] or [LLiu1]) that

$$
\mathcal{E}_{K_{0}}=\left\{E_{1}, \ldots, E_{n}, T-E_{1}, \ldots, T-E_{n}\right\} .
$$

Indeed, $e \cdot E_{i}=c_{i}$ and $e \cdot\left(U-E_{i}\right)=a-c_{i}$, both of which are positive because $e$ is reduced.

Property 4. Consider symplectic forms $\omega_{\epsilon}=U+T-\sum \epsilon E_{i}$. Their canonical class is $K_{0}=-2 U+(2 g-2) T+\sum_{i} E_{i}$, and for $\epsilon$ small, $\omega_{\epsilon} \cdot e>0$ for any reduced class $e=a U+b T-\sum_{i} c_{i} E_{i}$. Therefore, $K_{0}$ is in $\mathcal{K}_{e}$.

Property 5. Suppose now $e=a U+b T-\sum_{i} c_{i} E_{i}$ is a reduced class with nonnegative square. Let $\tau$ be the class of a symplectic form which is positive on $e$. Since both $a$ and $b$ are non-negative and one of them is positive, by the light cone lemma, $\tau$ must have a positive $U$ term as well. Therefore any symplectic canonical class in $\mathcal{K}_{e}$ is of the form $K=-2 U+b T+\sum s_{i} E_{i}$ with $s_{i}$ being odd. Since $K^{2}=8-8 g-n$, $b=2 g-2-\left[\left(\sum_{i} c_{i}^{2}-n\right) / 4\right]$. Thus we have $K_{0}-K=\left[\left(\sum_{i} s_{i}^{2}-n\right) / 4\right] T+\left(1-s_{i}\right) E_{i}$, and consequently

$$
\begin{aligned}
\left(K_{0}-K\right) \cdot e & =a \sum_{i}\left(s_{i}^{2}-1\right) / 4+\sum_{i}\left(1-s_{i}\right) \\
& =\sum_{i}\left(1-s_{i}\right)\left[\frac{\left(3-s_{i}\right)+(1-a)\left(1+s_{i}\right)}{4}\right]
\end{aligned}
$$

Let $S_{i}=\left(1-s_{i}\right)\left[\left(3-s_{i}\right)+(1-a)\left(1+s_{i}\right)\right]$. We will show that $S_{i} \geq 0$ for each $i$. When $s_{i} \geq 3$, the two factors of $S_{i}$ are both non-positive, so $S_{i}$ is non-negative. When $s_{i}=1$, $S_{i}=0$. Finally, when $s_{i} \leq-1$, the two factors are both non-negative, and therefore $S_{i}$ is non-negative. We have finished the proof of property 5 for a non-minimal ruled manifold and hence the proof of Lemma 3.4. 
We will now prove a rather general result relating the symplectic genus and the minimal genus of a reduced class, using Taubes' equivalence between Seiberg-Witten invariants and Gromov-Taubes invariants ([T2]). Let us first provide some background of this equivalence (see e.g. [LLiu1] and [T2]).

Recall that Seiberg-Witten invariants are defined on Spin ${ }^{c}$ structures. For manifolds without torsion-free homology group, like rational and irrational ruled manifolds, the Spinc structures correspond to characteristic classes. For this reason, we will simply speak of the Seiberg-Witten invariants of the characteristic classes. Suppose $K$ is a symplectic canonical class, then any class of the form $-K+2 e$ is a characteristic class. The Seiberg-Witten invariant of $-K+2 e$ is defined when its Seiberg-Witten dimension $-K \cdot e+e \cdot e$ is non-negative. For manifolds with $b^{+}=1$, the Seiberg-Witten invariants also depend on the chambers. In the presence of a symplectic form $\omega$, there is an $\omega$-symplectic chamber. On such a manifold, the Gromov-Taubes invariant of a class $e$ is a suitable count of $\omega$-symplectic surfaces representing $e$. The surface is not required to be connected, but is required to be embedded and any component with negative square is a $\omega$-symplectic sphere with square -1 .

When $K$ is the symplectic canonical class of $\omega$, a fundamental theorem of Taubes states that, if the Seiberg-Witten invariant of $-K+2 e$ in the $\omega$-symplectic chamber is nontrivial, then (i) $e$ is represented by a $J$-holomorphic curve (possibly singular) for any $\omega$-compatible almost complex structure $J$; (ii) the Seiberg-Witten invariant is the same as the Gromov-Taubes invariant of $e$ provided $e \cdot E \geq 0$ for any $E \in \mathcal{E}_{K}$.

PROPOSITION 3.5. Let $M$ be a non-minimal rational or irrational ruled manifold with a standard decomposition and a standard basis. Suppose $e$ is a reduced class. If $e \cdot e$ is no less than $\eta(e)-1$, then $e \cdot e \geq 0$ and $e$ is represented by a symplectic surface. Moreover, if $e$ is either a class of positive square or a primitive class with square $0, e$ is represented by a connected symplectic surface, and therefore its minimal genus is given by its symplectic genus.

Proof. We will first prove that $e$ is represented by a symplectic surface. By the definition of the symplectic genus and Lemma 3.4(4)

$$
K_{0} \cdot e+e \cdot e \leq 2 \eta(e)-2
$$

Therefore, under our assumption, the Seiberg-Witten dimension of the class $-K_{0}+2 e$ satisfies

$$
-K_{0} \cdot e+e \cdot e \geq 2(e \cdot e+1-\eta(e)) \geq 0 .
$$

Now we divide the proof into two cases.

In the case of rational manifold, for a symplectic from $\omega$ with $-K_{0}=3 H-\sum_{i} E_{i}$ as its canonical class, it is shown in [LLiu2] that $H$ is represented by an embedded $J$-holomorphic sphere for a generic almost complex structure $J$ compatible with $\omega$. Since the reduced class $e=a H-\sum b_{i} E_{i}$ has a positive $a$ term, $\left(K_{0}-e\right)$ has a negative $a$ term and so $\left(K_{0}-e\right) \cdot H<0$. Therefore, $K_{0}-e$ is not represented by a $J$-holomorphic curve, because the intersection number of two distinct $J$-holomorphic curves is non-negative. So the Seiberg-Witten invariant of $-K_{0}+2\left(K_{0}-e\right)=K_{0}-2 e$ is trivial by the result of Taubes. By the symmetry of Seiberg-Witten invariants (see Lemma 2.3 in [LLiu1]), the Seiberg-Witten invariant of $-K_{0}+2 e$ in the non-symplectic chamber is trivial. By the wall crossing formula of Seiberg-Witten invariants (see [KM] and Lemma 3.3 in [LLiu1]), the Seiberg-Witten invariant $S W_{\omega}\left(-K_{0}+2 e\right)$ in the $\omega$-symplectic chamber is non-trivial. Since $e$ is reduced, by Lemma 2.3(3), we have 
$e \cdot E \geq 0$ for any $E \in \mathcal{E}_{K_{0}}$. Thus, $e$ is represented by an embedded symplectic surface by the result of Taubes.

In the case of irrational ruled manifold, by [LLiu2], for any symplectic form $\omega$ with $K_{0}$ as its canonical class, $T$ is represented by a $J$-holomorphic sphere for a generic $\omega$-compatible almost complex structure $J$. Since a reduced class has a positive $U$ term and $U \cdot T=1$, we can show that $K_{0}-e$ has trivial Seiberg-Witten invariant in the $\omega$-symplectic chamber. Applying Lemma 2.3 and Lemma 3.3 in [LLiu1] as above, and notice that $-K_{0}+2 e$ has a positive $U$ term and that the class $\gamma$ in Lemma 3.3 in [LLiu1] is just the class $T$ here, we find that the Seiberg-Witten invariant of $-K_{0}+2 e$ is nontrivial. Taubes's result and Lemma 2.3(3) then can be applied to show that $e$ is represented by an embedded symplectic surface.

We have shown that $e$ is represented by a symplectic surface. This surface may have many components. Any component with negative square is a symplectic sphere with square -1 . However, since $e \cdot E \geq 0$ for any $E \in \mathcal{E}_{K_{0}}$, no such component exists. Thus, $e$ is represented by a symplectic surface the components of which all have non-negative square, and therefore $e \cdot e$ is non-negative. If $e \cdot e>0$, there can only be one component by the light cone lemma. If $e \cdot e=0$, again by the light cone lemma, there might be several components, all of which are multiples of the same class. All the multiplicities have to be one because of the adjunction formula. Thus, if $e$ is primitive, there is only one component. The proof is complete.

Notice that, as an immediate consequence of Proposition 3.5, the symplectic genus of certain reduced class is non-negative. In fact, this weaker assertion holds in much greater generality.

LEMMA 3.6. Let $M$ be a non-minimal rational or irrational ruled manifold with a standard decomposition and a standard basis.

1. The symplectic genus of any class with positive square or a primitive class with square 0 is non-negative.

2. Any class with square -1 or -2 has non-negative symplectic genus. In addition, the classes which are equivalent to reduced classes have positive symplectic genus, and those which are not equivalent to reduced classes have symplectic genus 0 .

Proof. Let $e$ be a class with square at least 0 and equivalent to a reduced class $e^{\prime}$. Due to Lemma 3.2(1), $e$ and $e^{\prime}$ have the same symplectic genus. Suppose that the symplectic genus of $e$ is negative, then $e \cdot e \geq-1 \geq \eta(e)-1$. By Proposition 3.5, $e^{\prime}$ is represented by a symplectic surface and hence the connected symplectic genus is non-negative. This is a contradiction.

When $e \cdot e=-1$, by Lemma 3.4(2), $e$ is either equivalent to a reduced class, or equivalent to $E_{1}, H-E_{1}-E_{2}$ or $T-E_{1}$. It is easy to see that $E_{1}, H-E_{1}-E_{2}$ or $T-E_{1}$ are all spherically representable and have symplectic genus zero. Suppose $e$ is a reduced class and $\eta(e) \leq 0$. Since $e \cdot e=-1$, it satisfies the assumption of Proposition 3.5, and we can conclude that $e \cdot e \geq 0$. This contradicts with our assumption. Therefore, by Lemma 3.2(1), any class equivalent to a reduced class has positive symplectic genus.

For the case of a class of square -2 , the same argument as in the previous paragraph proves that the symplectic genus can not be smaller than zero. What we still need to show is that there does not exist any reduced class $e$ with symplectic genus 0 . Suppose $e$ is such a class. Then by definition there is a $K \in \mathcal{K}$ such that $K \cdot e=0$. In light of Lemma 3.4(4), it is also necessary that $K_{0} \cdot e \leq 0$. We first exclude the case $K_{0} \cdot e<0$. Let $K^{\prime}$ be a symplectic canonical class such that $\mathcal{C}_{K^{\prime}}$ and $\mathcal{C}_{K_{0}}$ are in 
the same component of the positive cone $\mathcal{P}$. Notice that the argument in the proof of Lemma 3.4(5) actually proves that $K^{\prime} \cdot e \leq K_{0} \cdot e$ for any reduced class $e$. Therefore all such $K^{\prime}$ satisfies $K^{\prime} \cdot e<0$. It is clear that any symplectic canonical class is either a $K^{\prime}$ or a $-K^{\prime}$. Thus, there is no symplectic canonical class $K$ satisfying $K \cdot e=0$. This contradiction leaves the case $K_{0} \cdot e=0$ as the only possibility. In this case, the reflection $R^{e}$ along $e$ preserves $\mathcal{E}_{K_{0}}$ since it preserves $K_{0}$. So, if $F \in \mathcal{E}_{K_{0}}$, then $F^{\prime}=R^{e}(F) \in \mathcal{E}_{K_{0}}$, and $F^{\prime}=F+(e \cdot F) e$. By [LLiu2], for any symplectic form $\omega \in \mathcal{C}_{K_{0}}, F$ and $F^{\prime}$ are both represented by smooth $J$-holomorphic spheres for some generic $\omega$-compatible almost complex structure $J$, we have $F \cdot F^{\prime} \geq 0$ by the positivity of intersection. This fact, together with Lemma 3.4(3), leads to the following contradiction

$$
-1=F^{\prime} \cdot F^{\prime}=F \cdot F^{\prime}+(e \cdot F) e \cdot F^{\prime} \geq 0 .
$$

The lemma is proved.

We are ready to prove Theorem $\mathrm{B}$. In fact, we will prove the following more general result.

Theorem B'. Let $M$ be a rational or irrational ruled four-manifold. Suppose $e$ is a class with square at least -1 , and in the case that $e$ has square one, we further assume that $e$ is a primitive class. Then its symplectic genus is non-negative and there is an algorithm to calculate its symplectic genus. Furthermore, if $e \cdot e \geq \eta(e)-1$, then $e$ is represented by a connected symplectic surface, and therefore its minimal genus coincides with its symplectic genus.

Proof. When $M$ is minimal, $M$ is either $C P^{2}, S^{2} \times S^{2}$ or an $S^{2}$-bundle over a Riemann surface. The minimal genus problem for these manifolds has been completely solved in [LiL3-4].

When $M$ is non-minimal, with a choice of a standard decomposition and a standard basis, we can define reduced classes. Suppose $e$ is a class satisfying the conditions of Theorem B'. By Lemma 3.4, under an algorithm, $e$ can be transformed to a reduced class $\tilde{e}$ or a class $e^{\prime}$ which can be represented by a symplectic sphere.

Since $e \cdot e=\tilde{e} \cdot \tilde{e}$, and $\eta(\tilde{e})=\eta(e)$ by Lemma 3.2(4), we see that $\tilde{e}$ satisfies the conditions of Proposition 3.5 and Lemma 3.6(1). By Lemma 3.4(4), the symplectic genus of $\tilde{e}$ can be calculated with a simple formula. And by Lemma 3.6(1), the symplectic genus of $\tilde{e}$ is non-negative. Finally, by Proposition 3.5, $\tilde{e}$ is represented by a connected symplectic surface. The proof of Theorem B' is complete.

Theorem A. Let $M$ be a smooth, closed oriented 4-manifold with non-empty symplectic cone and $b^{+}(M)=1$. Then the symplectic genus of any class of positive square is non-negative, and it coincides with the minimal genus for any sufficiently large multiple of such a class.

Proof. In the rational and irrational ruled cases, by Theorem B', every class with positive square has positive symplectic genus. If $M$ is neither rational nor irrational ruled, we examine the minimal case first. Given a class $e$ with positive square and a symplectic form $\omega$, by the light cone lemma, either $\omega \cdot e>0$ or $-\omega \cdot e>0$. Let us assume that we are in the first situation. By a result in [Liu], $K(\omega) \cdot \omega \geq 0$ if $K$ is the canonical class of a symplectic form $\omega$. Then, by the light cone lemma, $K(\omega) \cdot e \geq 0$. Thus it follows directly from inequality (3.2) that the symplectic genus of $e$ is positive. For the non-minimal case, we claim that one can find $K \in \mathcal{K}_{e}$ such that $K \cdot e \geq 0$ if $e \cdot e \geq 0$. The positivity of $\eta(e)$ then follows immediately from it. Suppose $M=N \# n \bar{C} P^{2}$ is the (unique) minimal reduction of $M$. Let $E_{1}, \ldots, E_{n}$ be 
the generators of $H^{2}$ of the $n \overline{C P}^{2}$. Write $e=e_{m}-r_{1} E_{1}-\ldots-r_{n} E_{n}$, where $e_{m}$ is the pull back of a class in $H^{2}(N ; \mathbf{Z})$ also denoted by $e_{m}$. Pick a symplectic form $\omega_{m}$ on $N$ such that $\omega_{m} \cdot e_{m}>0$. Let $K_{m}$ be a symplectic canonical class of $\omega_{m}$. Then, as above, we have $e_{m} \cdot K_{m} \geq 0$. By the blow up construction, for sufficiently small $\epsilon$, the class $\left[\omega_{m}\right]-\epsilon E_{1}-\ldots-\epsilon E_{n}$ is realized by a symplectic form on $M$ with symplectic canonical class $K_{m}+E_{1}+\ldots+E_{n}$. Applying the reflections along the $E_{i}$, we see that $\left[\omega_{m}\right] \pm \epsilon E_{1} \pm \ldots \pm \epsilon E_{n}$ are realized by symplectic forms with symplectic canonical classes $K_{m} \mp E_{1} \mp \ldots, \mp E_{n}$. For possibly smaller $\epsilon$, the pairing between $e$ and $\left[\omega_{m}\right] \pm \epsilon E_{1} \pm \ldots \pm \epsilon E_{n}$ is positive. Therefore, any symplectic canonical class of the form $K=K_{m} \pm E_{1} \pm \ldots \pm E_{n}$ is in $\mathcal{K}_{e}$. Since $K_{m} \cdot e_{m} \geq 0$, by choosing $E_{i}$ or $-E_{i}$ appropriately, we can easily find a $K \in \mathcal{K}_{e}$ such that $K \cdot e \geq 0$.

The last statement of the theorem (for a class $e$ satisfying $e \cdot E \neq 0$ for any $E \in \mathcal{E}$ ) is a direct consequence of the following two results, together with Lemma 3.2(1). One result is in [LLiu1] that

$$
C_{M}=\left\{e \in \mathcal{P}|0<| e \cdot E \mid \text { for all } E \in \mathcal{E}_{M}\right\} .
$$

The other is due to Donaldson (see [D]). It states that, for any sufficiently large integer $N, N[\omega]$ can be represented by connected symplectic submanifolds. Now suppose that $e \cdot E=0$ for some $E \in \mathcal{E}$. By the result in [L1], there exists a symplectic form $\omega$ such that $E$ is represented by an $\omega$-symplectic sphere. Blowing down that sphere, we obtain a new symplectic manifold $M^{\prime}$. There is a class $e^{\prime}$ in $M^{\prime}$ which is pulled back to $e$. It is easy to see that $m\left(l e^{\prime}\right) \geq m(l e)$ and $\eta\left(l e^{\prime}\right)=\eta(l e)$ for any integer $l$. If $e^{\prime} \cdot E^{\prime} \neq 0$ for any $E^{\prime} \in \mathcal{E}_{M^{\prime}}$, then $\eta\left(l e^{\prime}\right)=m\left(l e^{\prime}\right)$ for sufficiently large $l$. Therefore $\eta(l e)=\eta\left(l e^{\prime}\right)=m\left(l e^{\prime}\right) \geq m(l e)$. Together with Lemma 3.2(1) we arrive at the conclusion that $\eta(l e)=m(l e)$. If there is still a class $E^{\prime} \in \mathcal{E}_{M^{\prime}}$ such that $e^{\prime} \cdot E^{\prime}=0$, we can continue the process above. However, this process can only be repeated finitely many times. The proof of Theorem A is complete.

We remark that, using some of the arguments in [LLiu1], in fact we are able to get an effective estimate on how large a multiple $N$ is allowed in the last statement of Theorem A. Here we just mention, in the case of a minimal manifold with $b^{+}=1$ which is neither rational nor irrational ruled, it suffices to take $N=2|e \cdot K| / e^{2}$, where $\pm K$ are the only two symplectic canonical classes. In particular, when a manifold with $b^{+}=1$ has a torsion symplectic canonical class, we are able to conclude that the minimal genus of every class $e$ with positive square coincides with its symplectic genus (which is simply $(e \cdot e) / 2+1)$. Such manifolds include the Enriques surface, hyperelliptic surfaces, any torus bundle over torus which has $b^{+}=1$. In addition, from the results in [LiL4], [Li1] and [Kr2], manifolds with the property that two genera coincide for any class of positive square include minimal irrational ruled manifold, rational manifold with $b^{-} \leq 9$ and the product of a circle with a fibered 3-manifold $Y$ with $b_{1}(Y)=1$.

We close this section with another remark. There are classes of positive square, which do not satisfy the conditions of Theorem B but still have the same symplectic genus and minimal genus. Some of them are actually represented by connected symplectic surfaces. For any positive integer $a$ bigger than 4, consider the reduced class $a H-\sum_{i=1}^{a^{2}-1} E_{i}$. Its square is 1 and symplectic genus $\left(a^{2}-3 a\right) / 2$. If we blow up $a^{2}-1$ points on a smooth curve of degree $a$, then the proper transform is a smooth curve in this given class. Others, including some classes in the non-trivial $S^{2}$-bundles over Riemann surfaces are not known to be represented by connected symplectic surfaces. To deal with such classes, we may need to find more constructive techniques as in [LiL3-4] and [Li1]. 
4. The classes represented by spheres. In this section we determine the set of classes represented by spheres and the orbits of $\operatorname{Diff}(M)$ on this set. We start with

Theorem C. Let $M$ be a rational or irrational ruled manifold and $e \in H^{2}(M)$ be a class with square at least -1 . If $\eta(e)=0$, then $\operatorname{PD}(e)$ is represented by a smoothly embedded sphere. Furthermore, if $\mathrm{PD}(e)$ is represented by a smoothly embedded sphere, then either $\eta(e)=0$ or $e$ is a non-primitive class of square zero with $e=p e^{\prime}$ and $\eta\left(e^{\prime}\right)=0$.

Proof. Suppose a standard decomposition and a standard basis are given. Let us first deal with the case $e \cdot e \geq 0$. Now suppose $m(e)=0$. Then by Lemma 3.2(1), $\eta(e) \leq 0$. By Lemma 3.6(1), $\eta(e)=0$ unless $e$ is a divisible class with square zero, ie. $e=p e^{\prime}$ for some $p \geq 2$ and some $e^{\prime}$ with $e^{\prime} \cdot e^{\prime}=0$. Since $\eta\left(p e^{\prime}\right)=\eta(e) \leq 0$, by Lemma 3.2(3), $\eta\left(e^{\prime}\right)$ can not be positive. In this case, by Lemmas 3.2(4) and 3.4(1), there is a reduced primitive class $\tilde{e}^{\prime}$ with the same square, the same symplectic genus and the same symplectic minimal genus as $e^{\prime}$. Since $\tilde{e}^{\prime}$ is primitive and reduced with $\tilde{e}^{\prime} \cdot \tilde{e}^{\prime}=0>\eta\left(\tilde{e}^{\prime}\right)-1$, we can apply Proposition 3.5 to conclude that $\eta\left(\tilde{e}^{\prime}\right)$ coincides with $m\left(\tilde{e}^{\prime}\right)$. Since $\eta\left(\tilde{e}^{\prime}\right) \leq 0$, and $m\left(\tilde{e}^{\prime}\right) \geq 0$ by definition, both of them are equal to zero. Therefore, in this case, $e^{\prime}$ has symplectic genus zero as well.

Suppose the symplectic genus $\eta(e)$ is zero. Again, there is a reduced class $\tilde{e}$ with the same square, the same divisibility, the same symplectic genus and the same symplectic minimal genus. Applying Proposition 3.5 to ẽ, together with Lemma 3.2(3), which excludes the case when $\tilde{e}$ is a divisible class with square zero, we conclude that $m(\tilde{e})=0$. Therefore, $m(e)$ is zero as well.

Finally we deal with the case that $e \cdot e=-1$. By Lemma 3.6(2), either $e$ has positive symplectic genus, or $\eta(e)=0$ and $e$ is spherically representable. When $\eta(e)>0, e$ is not spherically representable by a sphere due to Lemma 3.2(1). Thus, $e$ is spherically representable if and only if $\eta(e)=0$. The proof is finished.

For the convenience of the proof of Theorem D, we state the following corollary.

Corollary 4.1. Let $M$ be a rational or irrational ruled 4-manifold. Suppose $e$ is a class with positive square or a primitive class with square zero, the following statements are equivalent:

1. $e$ is represented by a smoothly embedded sphere.

2. $\eta(e)$ is zero.

3. $e$ is represented by a symplectically embedded sphere with respect to some symplectic form.

Proof. The equivalence of the first two statements follows from Theorem C. The equivalence of last two statements follows directly from Proposition 3.5 and Lemma 3.4(1).

We remark that Corollary 4.1 holds for classes with square -1 and -2 as well.

Having determined the set $\mathcal{S P \mathcal { H }} \mathcal{H}_{\geq-1}(M)$, we are going to classify the orbits of $\operatorname{Diff}(M)$ on this set. We begin with the difficult case when $M$ is rational.

THEOREM 4.2. Let $M$ be a rational manifold with a standard decomposition and a standard basis. Then the following classes are spherically representable:

1. $2 H$,

2. $(k+1) H-k E_{1}, k \geq 0$,

3. $(k+1) H-k E_{1}-E_{2}, k \geq 1$,

4. $k H-k E_{1}, k \geq 1$. 
Moreover, up to diffeomorphisms, any spherically representable class with nonnegative squares is equivalent to one of the above.

Proof. The first claim is well known. We just give a sketch here. $H$ and the $E_{i}$ are all spherically representable. Moreover, the spheres representing them can be chosen to be pairwisely disjoint. The first claim now follows from the elementary fact: if $A_{1}$ and $A_{2}$ are represented by two spheres which intersect at most at one point, then $A_{1}+A_{2}$ is spherically representable.

To prove the last claim, we need the following two results.

Proposition 4.3. Up to automorphisms of $H^{2}$, the set of spherically representable classes with non-negative square are given as above.

LEMMA 4.4. Let $\omega$ be a symplectic form with symplectic canonical class $K$.

1. Any class $R$ with positive square and represented by an $\omega$-symplectic sphere is in $\hat{\mathcal{C}}_{K}$ and satisfies $R \cdot(-K)>0$.

2. Any $R$ with square 0 and represented by an $\omega$-symplectic sphere is in $\overline{\mathcal{C}_{K}} \cap \mathcal{B}$ and satisfies $R \cdot(-K)>0$.

Proof of Proposition 4.3. The classes in the first three cases have positive square. In this case, the claim was proved by Kikuchi in [K1]. For classes of square 0, this was implicitly shown in [Li2]. In fact, it was shown in [Li2] that if $y$ is a primitive class represented by an embedded sphere, then there exists $x, z_{1}, \ldots, z_{n-1}$ such that

$$
H^{2}(M ; \mathbf{Z})=\left(\begin{array}{ll}
0 & 1 \\
1 & t
\end{array}\right) \oplus(n-1)(-1)
$$

with respect to the basis $\left\langle y, x, z_{1}, \ldots, z_{n-1}\right\rangle$. If $t$ is odd, let $\tilde{x}=x-[(t-1) / 2] y$, $\tilde{z}_{i}=z_{i}$ for $i=1, \ldots, n-1$; if $t$ is even, let $\tilde{x}=x-[(t-2) / 2] y+z_{1}, \tilde{z}_{1}=z_{1}+y, \tilde{z}_{i}=z_{i}$ for $i=2, \ldots, n-1$. Then, with respect to the new basis $\left\langle y, \tilde{x}, \tilde{z}_{1}, \ldots, \tilde{z}_{n-1}\right\rangle$,

$$
H^{2}(M ; \mathbf{Z})=\left(\begin{array}{ll}
0 & 1 \\
1 & 1
\end{array}\right) \oplus(n-1)(-1)
$$

Since $H^{2}(M ; \mathbf{Z})$ has the same decomposition with respect to the basis $<H-E_{1}, H, E_{2}, \ldots, E_{n}>$, there is an automorphism of $H^{2}(M ; \mathbf{Z})$ sending $y$ to $H-E_{1}$. The non-primitive case follows immediately.

Proof of Lemma 4.4. For any $\omega$-compatible almost complex structure $J$, the $\omega$-symplectic sphere representing $R$ can be taken $J$-holomorphic. Moreover, for a generic $\omega$-compatible almost complex structure $J$, any $E \cdot \mathcal{E}_{K}$ is represented by a smooth $J$-holomorphic sphere. Then $R \cdot E \geq 0$ for any $E \in \mathcal{E}_{K}$ by the positivity of intersection of pseudo-holomorphic curves. Thus, when $R$ has positive square, it is $\hat{\mathcal{C}}_{K}$, and when $R$ has square zero, it is in the $\overline{\mathcal{C}_{K}} \cap \mathcal{B}$. In either case, by the adjunction formula, $R \cdot(-K)=2+R \cdot R \geq 2$. The lemma is proved.

We now continue the proof of Theorem 4.2. Suppose $R$ is a class with nonnegative square and represented by a sphere. Let $R^{\prime}$ be a class in the list of Theorem 4.2 with the same square and the same divisibility. By Proposition 4.3 , there exists an automorphism $\phi$ such that $\phi^{*}(R)=R^{\prime}$. By Corollary 4.1, both $R$ and $R^{\prime}$ are represented by symplectic spheres (possibly with respect to different symplectic forms). By Lemma 4.4 and Theorem 2.8, we see that $\phi$ is realized by an orientation-preserving diffeomorphism. The proof of Theorem 4.2 is complete. 
We remark that, for a rational manifold with $b^{-} \leq 9$, Theorem 4.2 has been proved in $[\mathrm{K}]$ and [Li1-2]. In fact, in this case, it follows immediately from the fact that every automorphism is realized by an orientation-preserving diffeomorphism. Kikuchi ([K2]) also conjectured that Theorem 4.2 would hold in general.

Now we are ready to prove Theorem D.

Proof of Theorem $D$. When $M$ is neither rational nor irrational ruled, $M$ has a unique minimal reduction $M=N \# n \overline{C P}^{2}$. Let $E_{1}, \ldots, E_{n}$ be the generators of the $\overline{C P}^{2}$. The only spherically representable classes with square at least -1 are $\pm E_{1}, \ldots, \pm E_{n}$. They are carried to each other by trivial automorphisms.

On a minimal irrational ruled manifold $M$, among all classes with square at least -1 , up to sign, there is a unique primitive class which is spherically representable (see [LiL4]). Since - Id is in $\mathrm{D}(M)$, again there is a unique orbit when the square and the divisibility are fixed.

A minimal rational manifold is either $C P^{2}$ or $S^{2} \times S^{2}$. Let $H$ be a generator of $H^{2}\left(C P^{2} ; \mathbf{Z}\right) . \pm H$ and $\pm 2 H$ are the only spherically representable classes, with square 1 and 4 respectively (see $[\mathrm{KM}])$. Since $-\mathrm{Id} \in \mathrm{D}\left(C P^{2}\right)$, there is only one orbit when the square is fixed. Let $x$ be the class represented by $S^{2} \times\{p t\}$ and $y$ be the class represented by $\{p t\} \times S^{2}$. For each even number $2 l$, there are four spherically representable classes with square $2 l: \pm(x+l y)$ and $\pm(l x+y)$ (see $[\mathrm{Ku}]$ ). Since -Id and the automorphism switching the two factors are in $\mathrm{D}(M)$, the uniqueness of the orbits for fixed square is obvious.

Finally, let us consider the non-minimal manifolds. In the rational case, suppose $M$ is given a minimal reduction $M=C P^{2} \# n \overline{C P}^{2}$ and a standard basis; and in the irrational case, suppose $M$ is given a minimal reduction of the form $S^{2} \times \Sigma_{h} \#(n-$ 1) $\overline{C P}^{2}$ and a standard basis. We first treat the case of negative square.

Proposition 4.5. $\operatorname{Diff}(M)$ has one orbit on $\mathcal{S P \mathcal { H }}-1(M)$ when $n=1$ and $n \geq 3$, in the exceptional case $n=2, \operatorname{Diff}(M)$ has two orbits, one ordinary and one characteristic. $\operatorname{Diff}(M)$ has one orbit on $\mathcal{S P \mathcal { H }} \mathcal{H}_{-2}(M)$ when $n=2$ and $n \geq 4$, in the exceptional case $n=3, \operatorname{Diff}(M)$ has two orbits, one ordinary and one characteristic.

Proof. This follows from Lemma 3.4(2) and Lemma 3.6(2).

For an irrational ruled manifold, the only spherically representable classes with non-negative square are $\pm k T$.

From Theorem $\mathrm{C}$ we can list all the possible orbits with non-negative square. For a given square, when there are more than one orbit, they are distinguished by divisibility.

$s=0: \operatorname{Diff}(M)$ has infinitely many orbits on $\mathcal{S P} \mathcal{H}_{s}(M)$, represented by $k\left(H-E_{1}\right)$,

$s \geq 1$ and odd: $\operatorname{Diff}(M)$ has one orbit on $\mathcal{S P} \mathcal{H}_{s}(M)$, represented by $[(s+1) / 2] H-$ $[(s-1) / 2] E_{1}$,

$s=2$ or $s \geq 6$ and even: $\operatorname{Diff}(M)$ has one orbit on $\mathcal{S P H}(M)$ if $l \geq 2$, represented by $[(s+2) / 2] H-([s / 2)] E_{1}-E_{2}$,

$s=4: \operatorname{Diff}(M)$ has one orbit on $\mathcal{S P \mathcal { H }}(M)$ if $l \leq 1$, represented by $2 H$; two orbits if $l \geq 2$, represented by $2 H$ and $3 H-2 E_{1}-E_{2}$.

Theorem $\mathrm{D}$ is thus proved for all cases. 


\section{REFERENCES}

[Bi] P. Biran, Symplectic packings in dimension 4, Geom. and Funct. Anal., 7 (1997), no.3, pp. 420-437.

[D] S. DonalDSon, Symplectic submanifolds and almost-complex geometry, J. Differential Geom., 44 (1996), no.4, pp. 666-705.

[FM1] R. Friedman ANd J. MoRgan, On the diffeomorphism types of certain algebraic surfaces, J. Differential Geom., 27 (1988), no.3, pp. 371-398.

[FM2] R. Friedman and J. Morgan, Algebraic surfaces and Seiberg-Witten invariants, J. Algebraic Geom., 6 (1997), no. 3, pp. 445-479.

[FS] R. Fintushel AND R. SteRn, Immersed spheres in 4-manifolds and the immersed Thom conjecture, Turkish J. Math., 19(2) (1995), pp. 145-157.

[G] H. Z. GAO, Representing homology classes of 4-manifolds, Topology and its Application, 52(2) (1993), pp. 109-120.

[K1] K. KIKUCHI, Positive 2-spheres in 4-manifolds of signature (1, n), Pacific J. Math., 160 (1993), pp. 245-258.

[K2] K. KIKUCHI, Personal communication.

[KM] P. KRONhEIMER AND T. Mrowka, The genus of embedded surfaces in the projective plane, Math. Res. Letters, 1 (1994), pp. 797-808.

[Kr1] P. Kronheimer, Embedded surfaces and gauge theory in three and four dimensions, Survey in differential geometry, Vol. III (Cambridge, MA, 1996), pp. 243-298, Int. Press, Boston, MA, 1998.

[Kr2] P. Kronheimer, Minimal genus in $S^{1} \times M^{3}$, Invent. Math., 135 (1999), no.1, pp. 45-61.

[Ku] K. KUGA, Representing homology classes of $S^{2} \times S^{2}$, Topology, 23 (1984), pp. 133-137.

[La1] T. LaWson, Smooth embeddings of 2-spheres in 4-manifolds, Expo. Math., 10 (1992), pp. 289-309.

[La2] T. Lawson, The minimal Genus problem, Expo. Math., 15(1997), pp. 385-431.

[L1] T. J. LI, Smoothly embedded spheres in symplectic four manifolds, Proc. Amer. Math. Soc., 127 (1999), no. 2, pp. 609-613.

[L2] T. J. LI, Fiber sums of Lefschetz fibrations, in preparation.

[Li1] B. H. LI, Representing nonnegative homology classes of $C P^{2} \# n \overline{C P}^{2}$ by minimal genus embeddings, Tran. Amer. Math. Soc., 352 (2000), no. 9., pp. 4155-4169.

[Li2] B. H. LI, Embeddings of surfaces in 4-manifolds (II), Chinese Science Bull., 36 (1991), pp. 2030-2033.

[LLiu1] T. J. Li AND A. K. Liv, Uniqueness of symplectic canonical class, surface cone and symplectic cone of 4-manifolds with $b^{+}=1$, to appear in Journal of Differential Geometry.

[LLiu2] T. J. Li AND A. K. Liv, Symplectic structures on ruled surfaces and a generalized adjunction inequality, Math. Res. Letters, 2 (1995), pp. 453-471.

[LiL2] B. H. Li AND T. J. LI, Smooth minimal genera for small negative classes in $C P^{2} \# n \overline{C P}^{2}$ when $n \leq 9$, preprint.

[LiL3] B. H. LI, T. J. LI, Minimal genus smooth embeddings in $S^{2} \times S^{2}$ and $C P^{2} \# n \overline{C P}^{2}$ with $n \leq 8$, Topology, 37 (1998), pp. 573-594.

[LiL4] B. H. LI, T. J. LI, Minimal genus embeddings of surfaces in $S^{2}$-bundles over surfaces, Math. Res. Lett., 4 (1997), pp. 379-394.

[Mc1] D. McDuff, The structure of rational and ruled symplectic 4-manifolds, J. Amer. Math. Soc., 3 (1990), no. 3, pp. 679-712.

[Mc2] D. MCDuFF, Lectures on Gromov invariants for symplectic 4-manifolds, With notes by Wladyslav Lorek, NATO Adv. Sci. Inst. Ser. C Math. Phys. Sci., 488, Gauge theory and symplectic geometry (Montreal, PQ, 1995), pp. 175-210, Kluwer Acad. Publ., Dordrecht, 1997.

[Mc3] D. McDuff, Immersed spheres in symplectic 4-manifolds, Ann. Inst. Fourier (Grenoble), 42 (1992), no.1-2, pp. 369-392.

[MS] D. MCDuff AND D. SAlamon, A survey of symplectic 4-manifolds with $b^{+}=1$, Proc. Gokovo conference, 1995, pp. 47-60.

[OS] P. Oszvath, Z. SzABo, The symplectic Thom conjecture, Ann. of Math., (2) 151 (2000), no. 1, pp. 93-124.

[T1] C.H. TAUBES, The Seiberg-Witten invariants and symplectic forms, Math. Research Letters, 1 (1994), pp. 809-822. 
[T2] C. TAUBES, The Seiberg-Witten invariants and the Gromov invariants, Math. Research Letters, 2 (1995), pp. 221-238.

[W] C. T. C. WALl, Diffeomorphisms of 4-manifolds, J. London Math. Soc., 39 (1964), pp. 131-140. 